\title{
A New Iteration Method for Nonexpansive Mappings and Monotone Mappings in Hilbert Spaces
}

\author{
Jong Soo Jung \\ Department of Mathematics, Dong-A University, Busan 604-714, South Korea \\ Correspondence should be addressed to Jong Soo Jung, jungjs@mail.donga.ac.kr
}

Received 5 October 2009; Accepted 20 December 2009

Academic Editor: Jong Kim

Copyright (C) 2010 Jong Soo Jung. This is an open access article distributed under the Creative Commons Attribution License, which permits unrestricted use, distribution, and reproduction in any medium, provided the original work is properly cited.

We introduce a new composite iterative scheme by the viscosity approximation method for nonexpansive mappings and monotone mappings in a Hilbert space. It is proved that the sequence generated by the iterative scheme converges strongly to a common point of set of fixed points of nonexpansive mapping and the set of solutions of variational inequality for an inversestrongly monotone mappings, which is a solution of a certain variational inequality. Our results substantially develop and improve the corresponding results of [Chen et al. 2007 and Iiduka and Takahashi 2005]. Essentially a new approach for finding the fixed points of nonexpansive mappings and solutions of variational inequalities for monotone mappings is provided.

\section{Introduction}

Let $H$ be a real Hilbert space and $C$ a nonempty closed convex subset of $H$. Recall that a mapping $f: C \rightarrow C$ is a contraction on $C$ if there exists a constant $k \in(0,1)$ such that $\|f(x)-f(y)\| \leq k\|x-y\|, x, y \in C$. We use $\Sigma_{C}$ to denote the collection of mappings $f$ verifying the above inequality. That is $\Sigma_{C}=\{f: C \rightarrow C \mid f$ is a contraction with constant $k\}$. A mapping $S: C \rightarrow C$ is called nonexpansive if $\|S x-S y\| \leq\|x-y\|, x, y \in C$; see $[1,2]$ for the results of nonexpansive mappings. We denote by $F(S)$ the set of fixed points of $S$; that is, $F(S)=\{x \in C: x=S x\}$.

Let $P_{C}$ be the metric projection of $H$ onto $C$. A mapping $A$ of $C$ into $H$ is called monotone if for $x, y \in C,\langle x-y, A x-A y\rangle \geq 0$. The variational inequality problem is to find a $u \in C$ such that

$$
\langle v-u, A u\rangle \geq 0
$$


for all $v \in C$; see [3-6]. The set of solutions of the variational inequality is denoted by $\mathrm{VI}(C, A)$. A mapping $A$ of $C$ into $H$ is called inverse-strongly monotone if there exists a positive real number $\alpha$ such that

$$
\langle x-y, A x-A y\rangle \geq \alpha\|A x-A y\|^{2}
$$

for all $x, y \in C$; see [7-9]. For such a case, $A$ is called $\alpha$-inverse-strongly monotone.

In 2005, Iiduka and Takahashi [10] introduced an iterative scheme for finding a common point of the set of fixed points of a nonexapnsive mapping and the set of solutions of the variational inequality for an inverse-strong monotone mapping as follows. For an $\alpha$ inverse-strongly monotone mapping $A$ of $C$ to $H$ and a nonexpansive mapping $S$ of $C$ into itself such that $F(S) \cap \operatorname{VI}(C, A) \neq \emptyset, x_{1}=x \in C,\left\{\alpha_{n}\right\} \subset[0,1)$, and $\left\{\lambda_{n}\right\} \subset[0,2 \alpha]$,

$$
x_{n+1}=\alpha_{n} x+\left(1-\alpha_{n}\right) S P_{C}\left(x_{n}-\lambda_{n} A x_{n}\right)
$$

for every $n \geq 1$. They proved that the sequence generated by (1.3) converges strongly to $P_{F(S) \cap V I}(C, A) x$ under the conditions on $\left\{\alpha_{n}\right\}$ and $\left\{\lambda_{n}\right\}: \lambda_{n} \in[c, d]$ for some $c, d$ with $0<c<$ $d<2 \alpha$,

$$
\lim _{n \rightarrow \infty} \alpha_{n}=0, \quad \sum_{n=1}^{\infty} \alpha_{n}<\infty, \quad \sum_{n=1}^{\infty}\left|\alpha_{n+1}-\alpha_{n}\right|<\infty, \quad \sum_{n=1}^{\infty}\left|\lambda_{n+1}-\lambda_{n}\right|<\infty .
$$

On the other hand, the viscosity approximation method of selecting a particular fixed point of a given nonexpansive mapping was proposed by Moudafi [11]. In 2004, in order to extend Theorem 2.2 of Moudafi [11] to a Banach space setting, $\mathrm{Xu}$ [12] considered the the following explicit iterative process. For $S: C \rightarrow C$ nonexpansive mappings, $f \in \Sigma_{C}$ and $\alpha_{n} \in(0,1)$,

$$
x_{n+1}=\alpha_{n} f\left(x_{n}\right)+\left(1-\alpha_{n}\right) S x_{n}, \quad n \geq 1 .
$$

Moreover, in [12], he also studied the strong convergence of $\left\{x_{n}\right\}$ generated by (1.5) as $n \rightarrow$ $\infty$ in either a Hilbert space or a uniformly smooth Banach space and showed that the strong $\lim _{n \rightarrow \infty} x_{n}$ is a solution of a certain variational inequality.

In 2007, Chen et al. [13] considered the following iterative scheme as the viscosity approximation method of (1.3). For an $\alpha$-inverse-strongly-monotone mapping $A$ of $C$ to $H$ and a nonexpansive mapping $S$ of $C$ into itself such that $F(S) \cap \operatorname{VI}(C, A) \neq \emptyset, f \in \Sigma_{C}, x_{0} \in C$, $\left\{\alpha_{n}\right\} \subset[0,1)$, and $\left\{\lambda_{n}\right\} \subset[0,2 \alpha]$,

$$
x_{n+1}=\alpha_{n} f\left(x_{n}\right)+\left(1-\alpha_{n}\right) S P_{C}\left(x_{n}-\lambda_{n} A x_{n}\right), \quad n \geq 0,
$$

and showed that the sequence $\left\{x_{n}\right\}$ generated by (1.6) converges strongly to a point in $F(S) \cap$ $\mathrm{VI}(C, A)$ under condition (1.4) on $\left\{\alpha_{n}\right\}$ and $\left\{\lambda_{n}\right\}$, which is a solution of a certain variational inequality.

In this paper, motivated by above-mentioned results, we introduce a new composite iterative scheme by the viscosity approximation method. For an $\alpha$-inverse-strongly monotone 
mapping $A$ of $C$ to $H$ and a nonexpansive mapping $S$ of $C$ into itself such that $F(S) \cap$ $\operatorname{VI}(C, A) \neq \emptyset, f \in \Sigma_{C}, x_{0} \in C,\left\{\alpha_{n}\right\} \subset[0,1)$, and $\left\{\lambda_{n}\right\} \subset[0,2 \alpha]$,

$$
\begin{gathered}
y_{n}=\alpha_{n} f\left(x_{n}\right)+\left(1-\alpha_{n}\right) S P_{C}\left(x_{n}-\lambda_{n} A x_{n}\right), \\
x_{n+1}=\left(1-\beta_{n}\right) y_{n}+\beta_{n} S P_{C}\left(y_{n}-\lambda_{n} A y_{n}\right), \quad n \geq 0 .
\end{gathered}
$$

If $\beta_{n}=0$, then the iterative scheme (1.7) reduces to the iterative scheme (1.6). Under condition (1.4) on the sequences $\left\{\alpha_{n}\right\}$ and $\left\{\lambda_{n}\right\}$ and appropriate condition on sequence $\left\{\beta_{n}\right\}$, we show that the sequence $\left\{x_{n}\right\}$ generated by (1.7) converges strongly to a point in $F(S) \cap \operatorname{VI}(C, A)$, which is a solution of a certain variational inequality. Using this result, we also obtain a strong convergence result for finding a common fixed point of a nonexpansive mapping and a strictly pseudocontractive mapping. Moreover, we investigate the problem of finding a common point of the set of fixed points of a nonexpansive mapping and the set of zeros of an inverse-strongly monotone mapping. The main results develop and improve the corresponding results of Chen et al. [13] and Iiduka and Takahashi [10]. We point out that the iterative scheme (1.7) is a new approach for finding the fixed points of nonexpansive mappings and solutions of variational inequalities for monotone mappings.

\section{Preliminaries and Lemmas}

Let $H$ be a real Hilbert space with inner product $\langle\cdot, \cdot\rangle$ and norm $\|\cdot\|$, and $C$ a closed convex subset of $H$. We write $x_{n} \rightarrow x$ to indicate that the sequence $\left\{x_{n}\right\}$ converges weakly to $x$. $x_{n} \rightarrow x$ implies that $\left\{x_{n}\right\}$ converges strongly to $x$. For every point $x \in H$, there exists a unique nearest point in $C$, denoted by $P_{C} x$, such that

$$
\left\|x-P_{C} x\right\| \leq\|x-y\|
$$

for all $y \in C$. $P_{C}$ is called the metric projection of $H$ to $C$. It is well known that $P_{C}$ satisfies

$$
\left\langle x-y, P_{C} x-P_{C} y\right\rangle \geq\left\|P_{C} x-P_{C} y\right\|^{2}
$$

for every $x, y \in H$. Moreover, $P_{C} x$ is characterized by the properties

$$
\begin{gathered}
\left\langle x-P_{C} x, P_{C} x-y\right\rangle \geq 0 \\
\|x-y\|^{2} \geq\left\|x-P_{C} x\right\|^{2}+\left\|y-P_{C} x\right\|^{2}
\end{gathered}
$$

for all $x \in H, y \in C$. In the context of the variational inequality problem, this implies that

$$
u \in \operatorname{VI}(C, A) \Longleftrightarrow u=P_{C}(u-\lambda A u), \quad \text { for any } \lambda>0 .
$$


We state some examples for inverse-strongly monotone mappings. If $A=I-T$, where $T$ is a nonexpansive mapping of $C$ into itself and $I$ is the identity mapping of $H$, then $A$ is $1 / 2$ inverse-strongly monotone and $\operatorname{VI}(C, A)=F(T)$. A mapping $A$ of $C$ into $H$ is called strongly monotone if there exists a positive real number $\eta$ such that

$$
\langle x-y, A x-A y\rangle \geq \eta\|x-y\|^{2}
$$

for all $x, y \in C$. In such a case, we say that $A$ is $\eta$-strongly monotone. If $A$ is $\eta$-strongly monotone and $\kappa$-Lipschitz continuous, that is, $\|A x-A y\| \leq \kappa\|x-y\|$ for all $x, y \in C$, then $A$ is $\eta / \kappa^{2}$-inverse-strongly monotone.

If $A$ is an $\alpha$-inverse-strongly monotone mapping of $C$ into $H$, then it is obvious that $A$ is $1 / \alpha$-Lipschitz continuous. We also have that for all $x, y \in C$ and $\lambda>0$,

$$
\begin{aligned}
\|(I-\lambda A) x-(I-\lambda A) y\|^{2} & =\|(x-y)-\lambda(A x-A y)\|^{2} \\
& =\|x-y\|^{2}-2 \lambda\langle x-y, A x-A y\rangle+\lambda^{2}\|A x-A y\|^{2} \\
& \leq\|x-y\|^{2}+\lambda(\lambda-2 \alpha)\|A x-A y\|^{2} .
\end{aligned}
$$

So, if $\lambda \leq 2 \alpha$, then $I-\lambda A$ is a nonexpansive mapping of $C$ into $H$. The following result for the existence of solutions of the variational inequality problem for inverse-strongly monotone mappings was given in Takahashi and Toyoda [14].

Proposition 2.1. Let $C$ be a bounded closed convex subset of a real Hilbert space and $A$ an $\alpha$-inversestrongly monotone mapping of $C$ into $H$. Then, $\operatorname{VI}(C, A)$ is nonempty.

A set-valued mapping $T: H \rightarrow 2^{H}$ is called monotone if for all $x, y \in H, f \in T x$ and $g \in T y$ imply $\langle x-y, f-g\rangle \geq 0$. A monotone mapping $T: H \rightarrow 2^{H}$ is maximal if the graph $G(T)$ of $T$ is not properly contained in the graph of any other monotone mapping. It is known that a monotone mapping $T$ is maximal if and only if for $(x, f) \in H \times H,\langle x-y, f-g\rangle \geq 0$ for every $(y, g) \in G(T)$ implies $f \in T x$. Let $A$ be an inverse-strongly monotone mapping of $C$ into $H$ and let $N_{C} v$ be the normal cone to $C$ at $v$, that is, $N_{C} v=\{w \in H:\langle v-u, w\rangle \geq$ 0 , for all $u \in C\}$, and define

$$
T v= \begin{cases}A v+N_{C} v, & v \in C, \\ \emptyset, & v \notin C .\end{cases}
$$

Then $T$ is maximal monotone and $0 \in T v$ if and only if $v \in \operatorname{VI}(C, A)$; see $[15,16]$.

We need the following lemmas for the proof of our main results.

Lemma 2.2 (see [17]). Let $\left\{s_{n}\right\}$ be a sequence of nonnegative real numbers satisfying

$$
s_{n+1} \leq\left(1-\lambda_{n}\right) s_{n}+\beta_{n,} \quad n \geq 0,
$$


where $\left\{\lambda_{n}\right\}$ and $\left\{\beta_{n}\right\}$ satisfy the following conditions:

(i) $\left\{\lambda_{n}\right\} \subset[0,1]$ and $\sum_{n=0}^{\infty} \lambda_{n}=\infty$ or, equivalently, $\prod_{n=0}^{\infty}\left(1-\lambda_{n}\right)=0$;

(ii) $\lim \sup _{n \rightarrow \infty} \beta_{n} / \lambda_{n} \leq 0$ or $\sum_{n=0}^{\infty}\left|\beta_{n}\right|<\infty$.

Then $\lim _{n \rightarrow \infty} s_{n}=0$.

Lemma 2.3 (see [1], demiclosedness principle). Let $H$ be a real Hilbert space, $C$ a nonempty closed convex subset of $H$, and $T: C \rightarrow E$ a nonexpansive mapping. Then the mapping $I-T$ is demiclosed on $C$, where $I$ is the identity mapping; that is, $x_{n} \rightarrow x$ in $E$ and $(I-T) x_{n} \rightarrow y$ imply that $x \in C$ and $(I-T) x=y$.

Lemma 2.4. In a real Hilbert space $H$, there holds the following inequality:

$$
\|x+y\|^{2} \leq\|x\|^{2}+2\langle y, x+y\rangle
$$

for all $x, y \in H$.

\section{Main Results}

In this section, we introduce a new composite iterative scheme for nonexpansive mappings and inverse-strongly monotone mappings and prove a strong convergence of this scheme.

Theorem 3.1. Let $C$ be a closed convex subset of a real Hilbert space $H$. Let $A$ be an $\alpha$-inversestrongly monotone mapping of $C$ to $H$ and $S$ a nonexpansive mapping of $C$ into itself such that $F(S) \cap \operatorname{VI}(C, A) \neq \emptyset$, and $f \in \Sigma_{C}$. Let $\left\{x_{n}\right\}$ be a sequence generated by

$$
\begin{gathered}
x_{0} \in C, \\
y_{n}=\alpha_{n} f\left(x_{n}\right)+\left(1-\alpha_{n}\right) S P_{C}\left(x_{n}-\lambda_{n} A x_{n}\right), \\
x_{n+1}=\left(1-\beta_{n}\right) y_{n}+\beta_{n} S P_{C}\left(y_{n}-\lambda_{n} A y_{n}\right), \quad n \geq 0,
\end{gathered}
$$

where $\left\{\lambda_{n}\right\} \subset[0,2 \alpha],\left\{\alpha_{n}\right\} \subset[0,1)$, and $\left\{\beta_{n}\right\} \subset[0,1]$. If $\left\{\alpha_{n}\right\},\left\{\lambda_{n}\right\}$ and $\left\{\beta_{n}\right\}$ satisfy the following conditions:

(i) $\lim _{n \rightarrow \infty} \alpha_{n}=0 ; \sum_{n=0}^{\infty} \alpha_{n}=\infty$;

(ii) $\beta_{n} \subset[0, a)$ for all $n \geq 0$ and for some $a \in(0,1)$;

(iii) $\lambda_{n} \in[c, d]$ for some $c$, $d$ with $0<c<d<2 \alpha$;

(iv) $\sum_{n=0}^{\infty}\left|\alpha_{n+1}-\alpha_{n}\right|<\infty ; \sum_{n=0}^{\infty}\left|\beta_{n+1}-\beta_{n}\right|<\infty ; \sum_{n=0}^{\infty}\left|\lambda_{n+1}-\lambda_{n}\right|<\infty$,

then $\left\{x_{n}\right\}$ converges strongly to $q \in F(S) \cap V I(C, A)$, which is a solution of the following variational inequality:

$$
\langle(I-f)(q), q-p\rangle \leq 0, \quad p \in F(S) \cap \operatorname{VI}(C, A) .
$$


Proof. Let $z_{n}=P_{C}\left(x_{n}-\lambda_{n} A x_{n}\right)$ and $w_{n}=P_{C}\left(y_{n}-\lambda_{n} A y_{n}\right)$ for every $n \geq 0$. Let $u \in F(S) \cap$ $\mathrm{VI}(C, A)$. Since $I-\lambda_{n} A$ is nonexpansive and $u=P_{C}\left(u-\lambda_{n} A u\right)$ from (2.5), we have

$$
\begin{aligned}
\left\|z_{n}-u\right\| & =\left\|P_{C}\left(x_{n}-\lambda_{n} A x_{n}\right)-P_{C}\left(u-\lambda_{n} A u\right)\right\| \\
& \leq\left\|\left(x_{n}-\lambda_{n} A x_{n}\right)-\left(u-\lambda_{n} A u\right)\right\| \\
& \leq\left\|x_{n}-u\right\| .
\end{aligned}
$$

Similarly we have $\left\|w_{n}-u\right\| \leq\left\|y_{n}-u\right\|$.

We divide the proof into several steps.

Step 1. We show that $\left\{x_{n}\right\}$ is bounded. In fact, since

$$
\begin{aligned}
\left\|y_{n}-u\right\| & =\left\|\alpha_{n}\left(f\left(x_{n}\right)-u\right)+\left(1-\alpha_{n}\right)\left(S z_{n}-u\right)\right\| \\
& \leq \alpha_{n}\left\|f\left(x_{n}\right)-u\right\|+\left(1-\alpha_{n}\right)\left\|z_{n}-u\right\| \\
& \leq \alpha_{n}\left\|f\left(x_{n}\right)-f(u)\right\|+\alpha_{n}\|f(u)-u\|+\left(1-\alpha_{n}\right)\left\|x_{n}-u\right\| \\
& \leq \alpha_{n} k\left\|x_{n}-u\right\|+\left(1-\alpha_{n}\right)\left\|x_{n}-u\right\|+\alpha_{n}\|f(u)-u\| \\
& =\left(1-(1-k) \alpha_{n}\right)\left\|x_{n}-u\right\|+\alpha_{n}\|f(u)-u\| \\
& \leq \max \left\{\left\|x_{n}-u\right\|, \frac{1}{1-k}\|f(u)-u\|\right\},
\end{aligned}
$$

we have

$$
\begin{aligned}
\left\|x_{n+1}-u\right\| & =\left\|\left(1-\beta_{n}\right)\left(y_{n}-u\right)+\beta_{n}\left(S w_{n}-u\right)\right\| \\
& \leq\left(1-\beta_{n}\right)\left\|y_{n}-u\right\|+\beta_{n}\left\|w_{n}-u\right\| \\
& \leq\left(1-\beta_{n}\right)\left\|y_{n}-u\right\|+\beta_{n}\left\|y_{n}-u\right\| \\
& \leq \max \left\{\left\|x_{n}-u\right\|, \frac{1}{1-k}\|f(u)-u\|\right\} .
\end{aligned}
$$

By induction, we get

$$
\left\|x_{n}-u\right\| \leq \max \left\{\left\|x_{0}-u\right\|, \frac{1}{1-k}\|f(u)-u\|\right\}, \quad n \geq 0 .
$$

This implies that $\left\{x_{n}\right\}$ is bounded and so $\left\{y_{n}\right\},\left\{z_{n}\right\},\left\{w_{n}\right\},\left\{A x_{n}\right\}$, and $\left\{A y_{n}\right\}$ are bounded. Moreover, since $\left\|S z_{n}-u\right\| \leq\left\|x_{n}-u\right\|$ and $\left\|S w_{n}-u\right\| \leq\left\|y_{n}-u\right\|,\left\{S z_{n}\right\}$ and $\left\{S w_{n}\right\}$ are also bounded. By condition (i), we also obtain

$$
\left\|y_{n}-S z_{n}\right\|=\alpha_{n}\left\|f\left(x_{n}\right)-S z_{n}\right\| \longrightarrow 0 \quad(\text { as } n \longrightarrow \infty)
$$


Step 2. We show that $\lim _{n \rightarrow \infty}\left\|x_{n+1}-x_{n}\right\|=0$. From (3.1), we have

$$
\begin{gathered}
y_{n}=\alpha_{n} f\left(x_{n}\right)+\left(1-\alpha_{n}\right) S z_{n} \\
y_{n-1}=\alpha_{n-1} f\left(x_{n-1}\right)+\left(1-\alpha_{n-1}\right) S z_{n-1}, \quad n \geq 1
\end{gathered}
$$

Simple calculations show that

$$
y_{n}-y_{n-1}=\left(1-\alpha_{n}\right)\left(S z_{n}-S z_{n-1}\right)+\left(\alpha_{n}-\alpha_{n-1}\right)\left(f\left(x_{n-1}\right)-S z_{n-1}\right)+\alpha_{n}\left(f\left(x_{n}\right)-f\left(x_{n-1}\right)\right) \text {. }
$$

Since

$$
\begin{aligned}
\left\|z_{n}-z_{n-1}\right\| & \leq\left\|\left(x_{n}-\lambda_{n} A x_{n}\right)-\left(x_{n-1}-\lambda_{n-1} A x_{n-1}\right)\right\| \\
& \leq\left\|\left(x_{n}-\lambda_{n} A x_{n}\right)-\left(x_{n-1}-\lambda_{n} A x_{n-1}\right)\right\|+\left|\lambda_{n-1}-\lambda_{n}\right|\left\|A x_{n-1}\right\| \\
& \leq\left\|x_{n}-x_{n-1}\right\|+\left|\lambda_{n-1}-\lambda_{n}\right|\left\|A x_{n-1}\right\|
\end{aligned}
$$

for every $n \geq 1$, we have

$$
\begin{aligned}
\left\|y_{n}-y_{n-1}\right\| \leq & \left(1-\alpha_{n}\right)\left\|z_{n}-z_{n-1}\right\|+\left|\alpha_{n}-\alpha_{n-1}\right|\left\|f\left(x_{n-1}\right)-S z_{n-1}\right\|+\alpha_{n} k\left\|x_{n}-x_{n-1}\right\| \\
\leq & \left(1-\alpha_{n}\right)\left(\left\|x_{n}-x_{n-1}\right\|+\left|\lambda_{n-1}-\lambda_{n}\right|\left\|A x_{n-1}\right\|\right) \\
& +\left|\alpha_{n}-\alpha_{n-1}\right|\left\|f\left(x_{n-1}\right)-S z_{n-1}\right\|+\alpha_{n} k\left\|x_{n}-x_{n-1}\right\| \\
& \leq\left(1-(1-k) \alpha_{n}\right)\left\|x_{n}-x_{n-1}\right\|+L_{1}\left|\lambda_{n-1}-\lambda_{n}\right|+M_{1}\left|\alpha_{n}-\alpha_{n-1}\right|
\end{aligned}
$$

for every $n \geq 1$, where $M_{1}=\sup \left\{\left\|f\left(x_{n}\right)-S z_{n-1}\right\|: n \geq 1\right\}$ and $L_{1}=\sup \left\{\left\|A x_{n}\right\|: n \geq 0\right\}$.

On the other hand, from (3.1) we have

$$
\begin{gathered}
x_{n+1}=\left(1-\beta_{n}\right) y_{n}+\beta_{n} S w_{n}, \\
x_{n}=\left(1-\beta_{n-1}\right) y_{n-1}+\beta_{n-1} S w_{n-1} .
\end{gathered}
$$

Also, simple calculations show that

$$
x_{n+1}-x_{n}=\left(1-\beta_{n}\right)\left(y_{n}-y_{n-1}\right)+\beta_{n}\left(S w_{n}-S w_{n-1}\right)+\left(\beta_{n}-\beta_{n-1}\right)\left(S w_{n-1}-y_{n-1}\right) .
$$

Since

$$
\begin{aligned}
\left\|w_{n}-w_{n-1}\right\| & \leq\left\|\left(y_{n}-\lambda_{n} A y_{n}\right)-\left(y_{n-1}-\lambda_{n-1} A y_{n-1}\right)\right\| \\
& \leq\left\|\left(y_{n}-\lambda_{n} A y_{n}\right)-\left(y_{n-1}-\lambda_{n} A y_{n-1}\right)\right\|+\left|\lambda_{n-1}-\lambda_{n}\right|\left\|A y_{n-1}\right\| \\
& \leq\left\|y_{n}-y_{n-1}\right\|+\left|\lambda_{n-1}-\lambda_{n}\right|\left\|A y_{n-1}\right\|
\end{aligned}
$$


for every $n \geq 1$, it follows that

$$
\begin{aligned}
\left\|x_{n+1}-x_{n}\right\| \leq & \left(1-\beta_{n}\right)\left\|y_{n}-y_{n-1}\right\|+\beta_{n}\left\|w_{n}-w_{n-1}\right\|+\left|\beta_{n}-\beta_{n-1}\right|\left\|S w_{n-1}-y_{n-1}\right\| \\
\leq & \left(1-\beta_{n}\right)\left\|y_{n}-y_{n-1}\right\|+\beta_{n}\left(\left\|y_{n}-y_{n-1}\right\|+\left|\lambda_{n-1}-\lambda_{n}\right|\left\|A y_{n-1}\right\|\right) \\
& +\left|\beta_{n}-\beta_{n-1}\right|\left\|S w_{n-1}-y_{n-1}\right\| \\
\leq & \left\|y_{n}-y_{n-1}\right\|+\left|\lambda_{n-1}-\lambda_{n}\right|\left\|A y_{n-1}\right\|+\left|\beta_{n}-\beta_{n-1}\right|\left\|S w_{n-1}-y_{n-1}\right\| .
\end{aligned}
$$

Substituting (3.11) into (3.15), we derive

$$
\begin{aligned}
\left\|x_{n+1}-x_{n}\right\| \leq & \left(1-(1-k) \alpha_{n}\right)\left\|x_{n}-x_{n-1}\right\|+L_{1}\left|\lambda_{n-1}-\lambda_{n}\right|+M_{1}\left|\alpha_{n}-\alpha_{n-1}\right| \\
& +\left|\lambda_{n-1}-\lambda_{n}\right|\left\|A y_{n-1}\right\|+\left|\beta_{n}-\beta_{n-1}\right|\left\|S w_{n-1}-y_{n-1}\right\| \\
\leq & \left(1-(1-k) \alpha_{n}\right)\left\|x_{n}-x_{n-1}\right\|+L_{2}\left|\lambda_{n-1}-\lambda_{n}\right|+M_{1}\left|\alpha_{n}-\alpha_{n-1}\right|+M_{2}\left|\beta_{n}-\beta_{n-1}\right|,
\end{aligned}
$$

where $L_{2}=\sup \left\{L_{1}+\left\|A y_{n}\right\|: n \geq 1\right\}$ and $M_{2}=\sup \left\{\left\|S w_{n}-y_{n}\right\|: n \geq 0\right\}$. From conditions (i) and (iv), it is easy to see that

$$
\begin{gathered}
\lim _{n \rightarrow \infty}(1-k) \alpha_{n}=0, \quad \sum_{n=0}^{\infty}(1-k) \alpha_{n}=\infty, \\
\sum_{n=0}^{\infty}\left(M_{1}\left|\alpha_{n+1}-\alpha_{n}\right|+M_{2}\left|\beta_{n+1}-\beta_{n}\right|+L_{2}\left|\lambda_{n+1}-\lambda_{n}\right|\right)<\infty .
\end{gathered}
$$

Applying Lemma 2.2 to (3.16), we have

$$
\left\|x_{n+1}-x_{n}\right\| \longrightarrow 0 \text { as } n \longrightarrow \infty .
$$

By (3.11), we also have that $\left\|y_{n+1}-y_{n}\right\| \rightarrow 0$ as $n \rightarrow \infty$.

Step 3. We show that $\lim _{n \rightarrow \infty}\left\|x_{n}-y_{n}\right\|=0$ and $\lim _{n \rightarrow \infty}\left\|x_{n}-S z_{n}\right\|=0$. Indeed, it follows that

$$
\begin{aligned}
\left\|x_{n+1}-y_{n}\right\| & =\beta_{n}\left\|S w_{n}-y_{n}\right\| \\
& \leq \beta_{n}\left(\left\|S w_{n}-S z_{n}\right\|+\left\|S z_{n}-y_{n}\right\|\right) \\
& \leq a\left(\left\|w_{n}-z_{n}\right\|+\left\|S z_{n}-y_{n}\right\|\right) \\
& \leq a\left(\left\|y_{n}-x_{n}\right\|+\left\|S z_{n}-y_{n}\right\|\right) \\
& \leq a\left(\left\|y_{n}-x_{n+1}\right\|+\left\|x_{n+1}-x_{n}\right\|+\left\|S z_{n}-y_{n}\right\|\right),
\end{aligned}
$$

which implies that

$$
\left\|x_{n+1}-y_{n}\right\| \leq \frac{a}{1-a}\left(\left\|x_{n+1}-x_{n}\right\|+\left\|S z_{n}-y_{n}\right\|\right) \text {. }
$$


Journal of Inequalities and Applications

Obviously, by (3.7) and Step 2, we have $\left\|x_{n+1}-y_{n}\right\| \rightarrow 0$ as $n \rightarrow \infty$. This implies that

$$
\left\|x_{n}-y_{n}\right\| \leq\left\|x_{n}-x_{n+1}\right\|+\left\|x_{n+1}-y_{n}\right\| \longrightarrow 0 \text { as } n \longrightarrow \infty
$$

By (3.7) and (3.21), we also have

$$
\left\|x_{n}-S z_{n}\right\| \leq\left\|x_{n}-y_{n}\right\|+\left\|y_{n}-S z_{n}\right\| \longrightarrow 0 \quad \text { as } n \longrightarrow \infty
$$

Step 4. We show that $\lim _{n \rightarrow \infty}\left\|x_{n}-z_{n}\right\|=0$. To this end, let $u \in F(S) \cap \operatorname{VI}(C, A)$. Then, by convexity of $\|\cdot\|^{2}$, we have

$$
\begin{aligned}
\left\|y_{n}-u\right\|^{2} & =\left\|\alpha_{n} f\left(x_{n}\right)+\left(1-\alpha_{n}\right) S z_{n}-u\right\|^{2} \\
& \leq \alpha_{n}\left\|f\left(x_{n}\right)-u\right\|^{2}+\left(1-\alpha_{n}\right)\left\|S z_{n}-u\right\|^{2} \\
& \leq \alpha_{n}\left\|f\left(x_{n}\right)-u\right\|^{2}+\left(1-\alpha_{n}\right)\left\|z_{n}-u\right\|^{2} \\
& \leq \alpha_{n}\left\|f\left(x_{n}\right)-u\right\|^{2}+\left(1-\alpha_{n}\right)\left[\left\|x_{n}-u\right\|^{2}+\lambda_{n}\left(\lambda_{n}-2 \alpha\right)\left\|A x_{n}-A u\right\|^{2}\right] \\
& \leq \alpha_{n}\left\|f\left(x_{n}\right)-u\right\|^{2}+\left\|x_{n}-u\right\|^{2}+\left(1-\alpha_{n}\right) c(d-2 \alpha)\left\|A x_{n}-A u\right\|^{2} .
\end{aligned}
$$

So we obtain

$$
\begin{aligned}
- & \left(1-\alpha_{n}\right) c(d-2 \alpha)\left\|A x_{n}-A u\right\|^{2} \\
& \leq \alpha_{n}\left\|f\left(x_{n}\right)-u\right\|^{2}+\left(\left\|x_{n}-u\right\|+\left\|y_{n}-u\right\|\right)\left(\left\|x_{n}-u\right\|-\left\|y_{n}-u\right\|\right) \\
& \leq \alpha_{n}\left\|f\left(x_{n}\right)-u\right\|^{2}+\left(\left\|x_{n}-u\right\|+\left\|y_{n}-u\right\|\right)\left\|x_{n}-y_{n}\right\| .
\end{aligned}
$$

Since $\alpha_{n} \rightarrow 0$ and $\left\|x_{n}-y_{n}\right\| \rightarrow 0$ by condition (i) and (3.21), we have $\left\|A x_{n}-A u\right\| \rightarrow 0(n \rightarrow$ $\infty)$. Moreover, from (2.2) we obtain

$$
\begin{aligned}
\left\|z_{n}-u\right\|^{2}= & \left\|P_{C}\left(x_{n}-\lambda_{n} A x_{n}\right)-P_{C}\left(u-\lambda_{n} A u\right)\right\|^{2} \\
\leq & \left\langle x_{n}-\lambda_{n} A x_{n}-\left(u-\lambda_{n} A u\right), z_{n}-u\right\rangle \\
= & \frac{1}{2}\left\{\left\|\left(x_{n}-\lambda_{n} A x_{n}\right)-\left(u-\lambda_{n} A u\right)\right\|^{2}+\left\|z_{n}-u\right\|^{2}\right. \\
& \left.\quad-\left\|\left(x_{n}-\lambda_{n} A x_{n}\right)-\left(u-\lambda_{n} A u\right)-\left(z_{n}-u\right)\right\|^{2}\right\} \\
\leq & \frac{1}{2}\left\{\left\|x_{n}-u\right\|^{2}+\left\|z_{n}-u\right\|^{2}-\left\|x_{n}-z_{n}\right\|^{2}\right. \\
& \left.\quad+2 \lambda_{n}\left\langle x_{n}-z_{n}, A x_{n}-A u\right\rangle-\lambda_{n}^{2}\left\|A x_{n}-A u\right\|^{2}\right\},
\end{aligned}
$$


and so

$$
\left\|z_{n}-u\right\|^{2} \leq\left\|x_{n}-u\right\|^{2}-\left\|x_{n}-z_{n}\right\|^{2}+2 \lambda_{n}\left\langle x_{n}-z_{n}, A x_{n}-A u\right\rangle-\lambda_{n}^{2}\left\|A x_{n}-A u\right\|^{2} .
$$

And hence

$$
\begin{aligned}
\left\|y_{n}-u\right\|^{2} \leq & \alpha_{n}\left\|f\left(x_{n}\right)-u\right\|^{2}+\left(1-\alpha_{n}\right)\left\|z_{n}-u\right\|^{2} \\
\leq & \alpha_{n}\left\|f\left(x_{n}\right)-u\right\|^{2}+\left\|x_{n}-u\right\|^{2}-\left(1-\alpha_{n}\right)\left\|x_{n}-z_{n}\right\|^{2} \\
& +2\left(1-\alpha_{n}\right) \lambda_{n}\left\langle x_{n}-z_{n}, A x_{n}-A u\right\rangle-\left(1-\alpha_{n}\right) \lambda_{n}^{2}\left\|A x_{n}-A u\right\|^{2} .
\end{aligned}
$$

Then we have

$$
\begin{aligned}
\left(1-\alpha_{n}\right)\left\|x_{n}-z_{n}\right\|^{2} \leq & \alpha_{n}\left\|f\left(x_{n}\right)-u\right\|^{2}+\left(\left\|x_{n}-u\right\|+\left\|y_{n}-u\right\|\right)\left(\left\|x_{n}-u\right\|-\left\|y_{n}-u\right\|\right) \\
& +2\left(1-\alpha_{n}\right) \lambda_{n}\left\langle x_{n}-z_{n}, A x_{n}-A u\right\rangle-\left(1-\alpha_{n}\right) \lambda_{n}^{2}\left\|A x_{n}-A u\right\|^{2} \\
\leq & \alpha_{n}\left\|f\left(x_{n}\right)-u\right\|^{2}+\left(\left\|x_{n}-u\right\|+\left\|y_{n}-u\right\|\right)\left\|x_{n}-y_{n}\right\| \\
& +2\left(1-\alpha_{n}\right) \lambda_{n}\left\langle x_{n}-z_{n}, A x_{n}-A u\right\rangle-\left(1-\alpha_{n}\right) \lambda_{n}^{2}\left\|A x_{n}-A u\right\|^{2}
\end{aligned}
$$

Since $\alpha_{n} \rightarrow 0,\left\|x_{n}-y_{n}\right\| \rightarrow 0$ and $\left\|A x_{n}-A u\right\| \rightarrow 0$, we get $\left\|x_{n}-z_{n}\right\| \rightarrow 0$. Also by (3.21), we have

$$
\left\|y_{n}-z_{n}\right\| \leq\left\|y_{n}-x_{n}\right\|+\left\|x_{n}-z_{n}\right\| \longrightarrow 0 \quad(n \longrightarrow \infty)
$$

Step 5. We show that $\lim \sup _{n \rightarrow \infty}\left\langle f(q)-q, y_{n}-q\right\rangle \leq 0$ for $q \in F(S) \cap \operatorname{VI}(C, A)$, where $q$ is a solution of the variational inequality

$$
\langle(I-f)(q), q-p\rangle \leq 0, \quad p \in F(S) \cap \operatorname{VI}(C, A)
$$

To this end, choose a subsequence $\left\{z_{n_{i}}\right\}$ of $\left\{z_{n}\right\}$ such that

$$
\limsup _{n \rightarrow \infty}\left\langle f(q)-q, S z_{n}-q\right\rangle=\lim _{i \rightarrow \infty}\left\langle f(q)-q, S z_{n_{i}}-q\right\rangle
$$

Since $\left\{z_{n_{i}}\right\}$ is bounded, there exists a subsequence $\left\{z_{n_{i j}}\right\}$ of $\left\{z_{n_{i}}\right\}$ which converges weakly to $z$. We may assume without loss of generality that $z_{n_{i}}-z$. Since $\left\|S z_{n_{i}}-z_{n_{i}}\right\| \leq\left\|S z_{n_{i}}-x_{n_{i}}\right\|+$ $\left\|x_{n_{i}}-z_{n_{i}}\right\| \rightarrow 0$ by Steps 4 and 5 , we have $S z_{n_{i}}-z$. Then we can obtain $z \in F(S) \cap \operatorname{VI}(C, A)$. Indeed, let us first show that $z \in \operatorname{VI}(C, A)$. Let

$$
T v= \begin{cases}A v+N_{C} v, & v \in C, \\ \emptyset, & v \notin C .\end{cases}
$$


Journal of Inequalities and Applications

Then $T$ is maximal monotone. Let $(v, w) \in G(T)$. Since $w-A v \in N_{C} v$ and $z_{n} \in C$, we have

$$
\left\langle v-z_{n}, w-A v\right\rangle \geq 0
$$

On the other hand, from $z_{n}=P_{C}\left(x_{n}-\lambda_{n} A x_{n}\right)$, we have $\left\langle v-z_{n}, z_{n}-\left(x_{n}-\lambda_{n} A z_{n}\right)\right\rangle \geq 0$ and hence

$$
\left\langle v-z_{n}, \frac{z_{n}-x_{n}}{\lambda_{n}}+A x_{n}\right\rangle \geq 0
$$

Therefore we have

$$
\begin{aligned}
\left\langle v-z_{n_{i}}, w\right\rangle \geq & \left\langle v-z_{n_{i}}, A v\right\rangle \\
\geq & \left\langle v-z_{n_{i}}, A v\right\rangle-\left\langle v-z_{n_{i}}, \frac{z_{n_{i}}-x_{n_{i}}}{\lambda_{n_{i}}}+A x_{n_{i}}\right\rangle \\
= & \left\langle v-z_{n_{i}}, A v-A x_{n_{i}}-\frac{z_{n_{i}}-x_{n_{i}}}{\lambda_{n_{i}}}\right\rangle \\
= & \left\langle v-z_{n_{i}}, A v-A z_{n_{i}}\right\rangle+\left\langle v-z_{n_{i}}, A z_{n_{i}}-A x_{n_{i}}\right\rangle \\
& -\left\langle v-z_{n_{i}}, \frac{z_{n_{i}}-x_{n_{i}}}{\lambda_{n_{i}}}\right\rangle \\
\geq & \left\langle v-z_{n_{i}}, A z_{n_{i}}-A x_{n_{i}}\right\rangle-\left\langle v-z_{n_{i}}, \frac{z_{n_{i}}-x_{n_{i}}}{\lambda_{n_{i}}}\right\rangle .
\end{aligned}
$$

Hence we have $\langle v-z, w\rangle \geq 0$ as $i \rightarrow \infty$. Since $T$ is maximal monotone, we have $z \in T^{-1} 0$ and hence $z \in \operatorname{VI}(C, A)$.

On the another hand, by Steps 3 and $4,\left\|z_{n}-S z_{n}\right\| \leq\left\|z_{n}-x_{n}\right\|+\left\|x_{n}-S z_{n}\right\| \rightarrow 0$. So, by Lemma 2.3, we obtain $z \in F(S)$ and hence $z \in F(S) \cap \operatorname{VI}(C, A)$. Then by (3.30) we have

$$
\begin{aligned}
\limsup _{n \rightarrow \infty}\left\langle f(q)-q, S z_{n_{i}}-q\right\rangle & =\lim _{i \rightarrow \infty}\left\langle f(q)-q, S z_{n_{i}}-q\right\rangle=\langle f(q)-q, z-q\rangle \\
& =\langle(I-f)(q), q-z\rangle \leq 0 .
\end{aligned}
$$

Thus, from (3.7) we obtain

$$
\begin{aligned}
\limsup _{n \rightarrow \infty}\left\langle f(q)-q, y_{n}-q\right\rangle & \leq \limsup _{n \rightarrow \infty}\left\langle f(q)-q, y_{n}-S z_{n}\right\rangle+\limsup _{n \rightarrow \infty}\left\langle f(q)-q, S z_{n}-q\right\rangle \\
& \leq \limsup _{n \rightarrow \infty}\|f(q)-q\|\left\|y_{n}-S z_{n}\right\|+\limsup _{n \rightarrow \infty}\left\langle f(q)-q, S z_{n}-q\right\rangle \\
& \leq 0 .
\end{aligned}
$$


Step 6. We show that $\lim _{n \rightarrow \infty}\left\|x_{n}-q\right\|=0$ for $q \in F(S) \cap \operatorname{VI}(C, A)$, where $q$ is a solution of the variational inequality

$$
\langle(I-f)(q), q-p\rangle \leq 0, \quad p \in F(S) \cap \operatorname{VI}(C, A)
$$

Indeed, from Lemma 2.4, we have

$$
\begin{aligned}
\left\|x_{n+1}-q\right\|^{2} \leq & \left\|y_{n}-q\right\|^{2}=\left\|\alpha_{n}\left(f\left(x_{n}\right)-q\right)+\left(1-\alpha_{n}\right)\left(S z_{n}-q\right)\right\|^{2} \\
\leq & \left(1-\alpha_{n}\right)\left\|S z_{n}-q\right\|^{2}+2 \alpha_{n}\left\langle f\left(x_{n}\right)-q, y_{n}-q\right\rangle \\
\leq & \left(1-\alpha_{n}\right)^{2}\left\|z_{n}-q\right\|^{2}+2 \alpha_{n}\left\langle f\left(x_{n}\right)-f(q), y_{n}-q\right\rangle+2 \alpha_{n}\left\langle f(q)-q, y_{n}-q\right\rangle \\
\leq & \left(1-\alpha_{n}\right)^{2}\left\|x_{n}-q\right\|^{2}+2 \alpha_{n} k\left\|x_{n}-q\right\|\left\|y_{n}-q\right\|+2 \alpha_{n}\left\langle f(q)-q, y_{n}-q\right\rangle \\
\leq & \left(1-\alpha_{n}\right)^{2}\left\|x_{n}-q\right\|^{2}+2 \alpha_{n} k\left\|x_{n}-q\right\|\left(\left\|y_{n}-x_{n}\right\|+\left\|x_{n}-q\right\|\right) \\
& +2 \alpha_{n}\left\langle f(q)-q, y_{n}-q\right\rangle \\
= & \left(1-2(1-k) \alpha_{n}\right)\left\|x_{n}-q\right\|^{2}+2 \alpha_{n} k\left\|y_{n}-x_{n}\right\|\left\|x_{n}-q\right\|+2 \alpha_{n}\left\langle f(q)-q, y_{n}-q\right\rangle \\
\leq & \left(1-\overline{\alpha_{n}}\right)\left\|x_{n}-q\right\|^{2}+\overline{\alpha_{n}} \overline{\beta_{n}}
\end{aligned}
$$

where

$$
\bar{\alpha}_{n}=2(1-k) \alpha_{n}, \quad \bar{\beta}_{n}=\frac{k B}{1-k}\left\|y_{n}-x_{n}\right\|+\frac{1}{1-k}\left\langle f(q)-q, y_{n}-q\right\rangle,
$$

and $B=\sup \left\{\left\|x_{n}-q\right\|: n \geq 0\right\}$. It is easily seen that $\bar{\alpha}_{n} \rightarrow 0, \sum_{n=1}^{\infty} \bar{\alpha}_{n}=\infty$, and $\lim \sup _{n \rightarrow \infty} \bar{\beta}_{n} \leq$ 0 . Thus by Lemma 2.2, we obtain $x_{n} \rightarrow q$. This completes the proof.

Remark 3.2. (1) Theorem 3.1 improves the corresponding results in Chen et al. [13] and Iiduka and Takahashi [10]. In particular, if $\beta_{n}=0$ and $f\left(x_{n}\right)=x$ is constant in (3.1), then Theorem 3.1 reduces to Theorem 3.1 of Iiduka and Takahashi [10].

(2) We obtain a new composite iterative scheme for a nonexpansive mapping if $A=0$ in Theorem 3.1 as follows (see also Jung [18]):

$$
\begin{gathered}
x_{0} \in C, \\
y_{n}=\alpha_{n} f\left(x_{n}\right)+\left(1-\alpha_{n}\right) S x_{n}, \\
x_{n+1}=\left(1-\beta_{n}\right) y_{n}+\beta_{n} S y_{n}, \quad n \geq 0 .
\end{gathered}
$$

As a direct consequence of Theorem 3.1, we have the following result. 
Corollary 3.3. Let $C$ be a closed convex subset of a real Hilbert space $H$. Let $A$ be an $\alpha$-inversestrongly monotone mapping of $C$ to $H$ such that $V I(C, A) \neq \emptyset$, and $f \in \Sigma_{C}$. Let $\left\{x_{n}\right\}$ be a sequence generated by

$$
\begin{gathered}
x_{0} \in C, \\
y_{n}=\alpha_{n} f\left(x_{n}\right)+\left(1-\alpha_{n}\right) P_{C}\left(x_{n}-\lambda_{n} A x_{n}\right), \\
x_{n+1}=\left(1-\beta_{n}\right) y_{n}+\beta_{n} P_{C}\left(y_{n}-\lambda_{n} A y_{n}\right), \quad n \geq 0,
\end{gathered}
$$

where $\left\{\lambda_{n}\right\} \subset[0,2 \alpha],\left\{\alpha_{n}\right\} \subset[0,1)$, and $\left\{\beta_{n}\right\} \subset[0,1]$. If $\left\{\alpha_{n}\right\},\left\{\lambda_{n}\right\}$, and $\left\{\beta_{n}\right\}$ satisfy the following conditions:

(i) $\lim _{n \rightarrow \infty} \alpha_{n}=0 ; \sum_{n=0}^{\infty} \alpha_{n}=\infty$,

(ii) $\beta_{n} \subset[0, a)$ for all $n \geq 0$ and for some $a \in(0,1)$,

(iii) $\lambda_{n} \in[c, d]$ for some $c, d$ with $0<c<d<2 \alpha$,

(iv) $\sum_{n=0}^{\infty}\left|\alpha_{n+1}-\alpha_{n}\right|<\infty ; \sum_{n=0}^{\infty}\left|\beta_{n+1}-\beta_{n}\right|<\infty ; \sum_{n=0}^{\infty}\left|\lambda_{n+1}-\lambda_{n}\right|<\infty$,

then $\left\{x_{n}\right\}$ converges strongly to $q \in \operatorname{VI}(C, A)$, which is a solution of the following variational inequality:

$$
\langle(I-f)(q), q-p\rangle \leq 0, \quad p \in V I(C, A) .
$$

\section{Applications}

In this section, as in $[10,13]$, we obtain two theorems in a Hilbert space by using Theorem 3.1. A mapping $T: C \rightarrow C$ is called strictly pseudocontractive if there exists $\alpha$ with $0 \leq \alpha<1$ such that

$$
\|T x-T y\|^{2} \leq\|x-y\|^{2}+\alpha\|(I-T) x-(I-T) y\|^{2}
$$

for every $x, y \in C$. If $\alpha=0$, then $T$ is nonexpansive. Put $A=I-T$, where $T: C \rightarrow C$ is a strictly pseudocontractive mapping with $\alpha$. Then $A$ is $(1-\alpha) / 2$-inverse-strongly monotone; see [7]. Actually, we have, for all $x, y \in C$,

$$
\|(I-A) x-(I-A) y\|^{2} \leq\|x-y\|^{2}+\alpha\|A x-A y\|^{2} .
$$

On the other hand, since $H$ is a real Hilbert space, we have

$$
\|(I-A) x-(I-A) y\|^{2}=\|x-y\|^{2}+A\|A x-A y\|^{2}-2\langle x-y, A x-A y\rangle .
$$

Hence we have

$$
\langle x-y, A x-A y\rangle \geq \frac{1-\alpha}{2}\|A x-A y\|^{2} .
$$


Using Theorem 3.1, we first get a strong convergence theorem for finding a common fixed point of a nonexpansive mapping and a strictly pseudocontractive mapping.

Theorem 4.1. Let $C$ be a closed convex subset of a real Hilbert space $H$. Let $T$ be an $\alpha$-strictly pseudocontractive mapping of $C$ into itself and $S$ a nonexpansive mapping of $C$ into itself such that $F(S) \cap F(T) \neq \emptyset$, and $f \in \Sigma_{C}$. Let $\left\{x_{n}\right\}$ be a sequence generated by

$$
\begin{gathered}
x_{0} \in C, \\
y_{n}=\alpha_{n} f\left(x_{n}\right)+\left(1-\alpha_{n}\right) S\left(\left(1-\lambda_{n}\right) x_{n}+\lambda_{n} T x_{n}\right), \\
x_{n+1}=\left(1-\beta_{n}\right) y_{n}+\beta_{n} S\left(\left(1-\lambda_{n}\right) y_{n}+\lambda_{n} T y_{n}\right), \quad n \geq 0,
\end{gathered}
$$

where $\left\{\lambda_{n}\right\} \subset[0,1-\alpha),\left\{\alpha_{n}\right\} \subset[0,1)$, and $\left\{\beta_{n}\right\} \subset[0,1]$. If $\left\{\alpha_{n}\right\},\left\{\lambda_{n}\right\}$, and $\left\{\beta_{n}\right\}$ satisfy the conditions:

(i) $\lim _{n \rightarrow \infty} \alpha_{n}=0 ; \sum_{n=0}^{\infty} \alpha_{n}=\infty$,

(ii) $\beta_{n} \subset[0, a)$ for all $n \geq 0$ and for some $a \in(0,1)$,

(iii) $\lambda_{n} \in[c, d]$ for some $c$, $d$ with $0<c<d<1-\alpha$,

(iv) $\sum_{n=0}^{\infty}\left|\alpha_{n+1}-\alpha_{n}\right|<\infty ; \sum_{n=0}^{\infty}\left|\beta_{n+1}-\beta_{n}\right|<\infty ; \sum_{n=0}^{\infty}\left|\lambda_{n+1}-\lambda_{n}\right|<\infty$,

then $\left\{x_{n}\right\}$ converges strongly to $q \in F(S) \cap F(T)$, which is a solution of the following variational inequality:

$$
\langle(I-f)(q), q-p\rangle \leq 0, \quad p \in F(S) \cap F(T) .
$$

Proof. Put $A=I-T$. Then $A$ is $(1-\alpha) / 2$-inverse-strongly monotone. We have $F(T)=\operatorname{VI}(C, A)$ and $P_{C}\left(x_{n}-\lambda_{n} A x_{n}\right)=\left(1-\lambda_{n}\right) x_{n}+\lambda_{n} T x_{n}$. Thus, the desired result follows from Theorem 3.1.

Using Theorem 3.1, we also have the following result.

Theorem 4.2. Let $H$ be a real Hilbert space $H$. Let $A$ be an $\alpha$-inverse-strongly monotone mapping of $H$ into itself and $S$ a nonexpansive mapping of $H$ into itself such that $F(S) \cap A^{-1} 0 \neq \emptyset$, and $f \in \Sigma_{C}$. Let $\left\{x_{n}\right\}$ be a sequence generated by

$$
\begin{gathered}
x_{0} \in H, \\
y_{n}=\alpha_{n} f\left(x_{n}\right)+\left(1-\alpha_{n}\right) S\left(x_{n}-\lambda_{n} A x_{n}\right), \\
x_{n+1}=\left(1-\beta_{n}\right) y_{n}+\beta_{n} S\left(y_{n}-\lambda_{n} A y_{n}\right), \quad n \geq 0,
\end{gathered}
$$

where $\left\{\lambda_{n}\right\} \subset[0,2 \alpha),\left\{\alpha_{n}\right\} \subset[0,1)$, and $\left\{\beta_{n}\right\} \subset[0,1]$. If $\left\{\alpha_{n}\right\},\left\{\lambda_{n}\right\}$, and $\left\{\beta_{n}\right\}$ satisfy the conditions:

(i) $\lim _{n \rightarrow \infty} \alpha_{n}=0 ; \sum_{n=0}^{\infty} \alpha_{n}=\infty$,

(ii) $\beta_{n} \subset[0, a)$ for all $n \geq 0$ and for some $a \in(0,1)$,

(iii) $\lambda_{n} \in[c, d]$ for some $c, d$ with $0<c<d<2 \alpha$,

(iv) $\sum_{n=0}^{\infty}\left|\alpha_{n+1}-\alpha_{n}\right|<\infty ; \sum_{n=0}^{\infty}\left|\beta_{n+1}-\beta_{n}\right|<\infty ; \sum_{n=0}^{\infty}\left|\lambda_{n+1}-\lambda_{n}\right|<\infty$, 
then $\left\{x_{n}\right\}$ converges strongly to $q \in F(S) \cap A^{-1} 0$, which is a solution of the following variational inequality:

$$
\langle(I-f)(q), q-p\rangle \leq 0, \quad p \in F(S) \cap A^{-1} 0 .
$$

Proof. We have $A^{-1} 0=V I(H, A)$. So, putting $P_{H}=I$, by Theorem 3.1, we obtain the desired result.

Remark 4.3. If $\beta_{n}=0$ in Theorems 4.1 and 4.2 , then Theorems 4.1 and 4.2 reduce to Chen et al. [13, Theorems 4.1 and 4.2]. Theorems 4.1 and 4.2 also extend in Iiduka and Takahashi [10, Theorems 4.1 and 4.2$]$ to the viscosity methods in composite iterative schemes.

\section{Acknowledgments}

This research was supported by Basic Science Research Program through the National Research Foundation of Korea (NRF) funded by the Ministry of Education, Science and Technology (2009-0064444). The author thanks the referees for their valuable comments and suggestions, which improved the presentation of this paper.

\section{References}

[1] K. Goebel and W. A. Kirk, Topics in Metric Fixed Point Theory, vol. 28 of Cambridge Studies in Advanced Mathematics, Cambridge University Press, Cambridge, UK, 1990.

[2] W. Takahashi, Nonlinear Functional Analysis: Fixed Point Theory and Its Applications, Yokohama Publishers, Yokohama, Japan, 2000.

[3] F. E. Browder, "Nonlinear monotone operators and convex sets in Banach spaces," Bulletin of the American Mathematical Society, vol. 71, pp. 780-785, 1965.

[4] R. E. Bruck Jr., "On the weak convergence of an ergodic iteration for the solution of variational inequalities for monotone operators in Hilbert space," Journal of Mathematical Analysis and Applications, vol. 61, no. 1, pp. 159-164, 1977.

[5] P. L. Lions and G. Stampacchia, "Variational inequalities," Communications on Pure and Applied Mathematics, vol. 20, pp. 493-517, 1967.

[6] W. Takahashi, "Nonlinear complementarity problem and systems of convex inequalities," Journal of Optimization Theory and Applications, vol. 24, no. 3, pp. 499-506, 1978.

[7] F. E. Browder and W. V. Petryshyn, "Construction of fixed points of nonlinear mappings in Hilbert space," Journal of Mathematical Analysis and Applications, vol. 20, pp. 197-228, 1967.

[8] H. Iiduka, W. Takahashi, and M. Toyoda, "Approximation of solutions of variational inequalities for monotone mappings," Panamerican Mathematical Journal, vol. 14, no. 2, pp. 49-61, 2004.

[9] F. Liu and M. Z. Nashed, "Regularization of nonlinear ill-posed variational inequalities and convergence rates," Set-Valued Analysis, vol. 6, no. 4, pp. 313-344, 1998.

[10] H. Iiduka and W. Takahashi, "Strong convergence theorems for nonexpansive mappings and inversestrongly monotone mappings," Nonlinear Analysis: Theory, Methods E Applications, vol. 61, no. 3, pp. 341-350, 2005.

[11] A. Moudafi, "Viscosity approximation methods for fixed-points problems," Journal of Mathematical Analysis and Applications, vol. 241, no. 1, pp. 46-55, 2000.

[12] H.-K. Xu, "Viscosity approximation methods for nonexpansive mappings," Journal of Mathematical Analysis and Applications, vol. 298, no. 1, pp. 279-291, 2004.

[13] J. Chen, L. Zhang, and T. Fan, "Viscosity approximation methods for nonexpansive mappings and monotone mappings," Journal of Mathematical Analysis and Applications, vol. 334, no. 2, pp. 1450-1461, 2007.

[14] W. Takahashi and M. Toyoda, "Weak convergence theorems for nonexpansive mappings and monotone mappings," Journal of Optimization Theory and Applications, vol. 118, no. 2, pp. 417-428, 2003. 
[15] R. T. Rockafellar, "On the maximality of sums of nonlinear monotone operators," Transactions of the American Mathematical Society, vol. 149, pp. 75-88, 1970.

[16] R. T. Rockafellar, "Monotone operators and the proximal point algorithm," SIAM Journal on Control and Optimization, vol. 14, no. 5, pp. 877-898, 1976.

[17] H.-K. Xu, "An iterative approach to quadratic optimization," Journal of Optimization Theory and Applications, vol. 116, no. 3, pp. 659-678, 2003.

[18] J. S. Jung, "Strong convergence on composite iterative methods for nonexpansive mappings," Journal of the Korean Mathematical Society, vol. 46, no. 6, pp. 1143-1156, 2009. 